Open Access : ISSN : 1848-7718

Original scientific paper

http://www.pub.iapchem.org/ojs/index.php/admet/index

\title{
Association of vancomycin with lipid vesicles
}

\author{
Xiaohui Hu and Kin Yip Tam * \\ Drug Development Core, Faculty of Health Sciences, University of Macau, Macau S.A.R., China \\ *Corresponding Author: E-mail: kintam@umac.mo; Tel.: +853-88224988; Fax: +835-88222314
}

Received: June 15, 2017; Revised: July 22, 2017; Published: September 29, 2017

\begin{abstract}
Antibiotics play a pivotal role in modern medicine for the treatment of bacterial infection in patients. Membrane defines the boundary between single cell and its environment and is a main target for antibacterial agents. To better understand the mechanism of antibiotics action on microbes, we utilized liposome as membrane mimic model to study antibiotics interaction with bacterial membrane by variety of biophysical methods. Isothermal calorimetry and fluorescence photometry experiments were performed to examine interaction between antibiotics and liposome. We found that vancomycin, one of the most important antibiotics for the treatment of serious infections by gram-positive bacteria, binds to the liposome. The association between the drug and the liposome does not involve the tail part of the lipids. Moreover, the binding affinity increases along with the increment of liposome size. Of three major lipid components, phosphatidylglycerol is the preferential target for vancomycin binding. We also showed that vancomycin associates with vesicle derived from Staphylococcus aureus membrane in a similar manner as the binding to liposome. Our data suggested that vancomycin associates with bacterial membrane through direct interaction with lipid head groups with the extent of the association depending very much on specific type of lipids and curvature of local membrane structure.
\end{abstract}

\section{Keywords}

Liposome; membrane; binding; phospholipids; antibiotics

\section{Introduction}

Pathogenic bacteria pose a major threaten to public health, especially in under-developed region of the world. Large scale production and use of antibiotic penicillin during the World War II is a hallmark of modern antimicrobial therapy. Since then, a plethora of antimicrobial agents have been developed and commercialized, playing significant roles in improvement of human health, highlighted by gradual increasing of average life span worldwide. Prevailing of antibiotic treatment improves human health on one hand, but also triggers resistance of microbes to the drugs [1,2].

Depending on their structures and target sites within microorganism, different antibacterial drugs have distinct modes of action [3]. Of them, related to cell membrane are: inhibition or block of cell wall synthesis, inhibition of membrane protein or lipid synthesis and interference of cell membrane function. Some antibacterial peptides [4,5], peptidomimics [6] and small proteins [7] could interact with or insert into cell membrane, where they interfere membrane formation or alter local membrane structure, thus reducing bacteria growth or reproduction. Bacterial membrane is surrounded by a layer of peptidoglycan cell wall. This unique feature had rendered great interest in searching for glycopeptide and 
lipoglycopeptide [8], which mimics bacterial cell wall synthesis substrates or binds to synthesis intermediates, thus inhibits cell wall synthesis, while having little effect on host cells.

Glycopeptide antibiotic vancomycin has been used to treat a number of infections caused by broad spectrum of gram-positive bacteria $[9,10]$. It inhibits cell wall formation by binding to D-Ala-D-Ala terminus of peptidoglycan precursors and preventing them from cross-linking $[11,12]$. To cope with rising of antibiotic resistance including vancomycin, development of novel anti-bacterial agents with multiple action modes had been a promising strategy [13-15]. Herein, we studied the binding property of vancomycin to artificial and bacterial membranes using different biophysics methods with the aim to obtain a better understanding of the binding process for expanding its antibiotic repository.

\section{Experimental}

\section{Materials}

1-palmitoyl-2-oleoyl-sn-glycero-3-phospho-(1'-rac-glycerol) (sodium salt) (POPG), 1-hexadecanoyl-2-(9Zoctadecenoyl)-sn-glycero-3-phosphoethanolamine (POPE), 1-oleoyl-2-[12-[(7-nitro-2-1,3-benzoxadiazol-4yl)amino]dodecanoyl]-sn-glycero-3-phosphocholine (NBD PC) and cardiolipin (CL) were purchased from Avanti Polar Lipids. Vancomycin and nafcillin were obtained from Sigma. Lysozyme and RNaseA were purchased from Merck. Staphylococcus aureus was from ATCC (strain \#43300) and cultured in tryptic soy broth medium (Becton, Dickinson and Company). Other chemicals were obtained from Sigma.

\section{Liposome preparation}

Lipids of known mass in chloroform were well-mixed, then dried at room temperature in a glass tube by continuous purging of high pure nitrogen gas. Being thoroughly dried in a speed vacuum, the lipids were re-

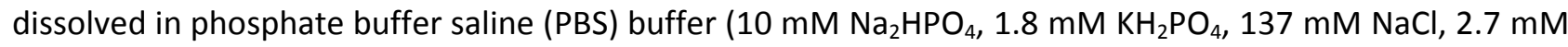
$\mathrm{KCl}, \mathrm{pH}$ 7.4) pre-warmed over $45^{\circ} \mathrm{C}$. After repeated pipetting, the hydrated lipids were applied to a Lipex extruder (Northern Lipids Inc.) equipped with polycarbonate membrane for more than 10 times. Scattering profile of the liposome was then measured on a BI-200SM goniometer (Brookhaven Instruments) while being excited by a laser passing $637 \mathrm{~nm}$ filter with 100 micron pinhole from 90 degree angle at $25{ }^{\circ} \mathrm{C}$. Data was collected and processed by the dynamic light scatter software coming with the equipment.

\section{Isolation of membrane from Staphylococcus aureus}

Isolation of membrane was carried out as described in the literature [16]. Briefly, bacteria were cultured till late exponential phase before harvest. Cells was then re-suspended in $50 \mathrm{mM}$ potassium phosphate buffer, pH 8.0 and digested by lysozyme, deoxyribonuclease-1 and ribonuclease for 30 minutes in the presence of $10 \mathrm{mM} \mathrm{MgSO}$ at $37{ }^{\circ} \mathrm{C}$. The reaction was stopped abruptly by $15 \mathrm{mM}$ EDTA for 1 minute. Additional $\mathrm{MgSO}_{4}$ was then added to the system to bring its final concentration back to $10 \mathrm{mM}$. Digested cells was centrifuged at room temperature, $25000 \mathrm{~g}$ for 45 minutes to pellet the membrane, which was then re-suspended in PBS and applied through 18-g needle for more than 20 times. Resultant membrane vesicle was quantified by measuring protein concentration (Pierce ${ }^{\mathrm{TM}}$ BCA protein assay, Thermo Scientific), assuming equivalent amount of protein and lipid. Size distribution of the vesicles was assessed on a BI200SM goniometer as describe in previous section.

\section{Isothermal calorimetry measurement}

In a typical isothermal calorimetry (ITC) experiment carried out on a MicroCal iTC200 calorimeter (Malvern Instruments), $50 \mathrm{mM}$ antibiotics were titrated into liposome with $5 \mathrm{mM}$ lipid (total or individual) in PBS buffer at $25^{\circ} \mathrm{C}$. After each injection, system was allowed to regain thermal balance between sample 
and reference chambers for 180 seconds with continuous stir of $750 \mathrm{rpm}$. Acquired data were processed by ITC-costumed Origin7 software using one-site model to avoid complication. Reference subtractions were performed against the data obtained by titrating antibiotic into PBS buffer.

\section{Fluorescence spectroscopy}

Spectra of the fluorescent lipid incorporated liposome were collected on an LS55 Fluorescence spectrometer (PerkinElmer) at room temperature. Fluorescent spectra of freshly prepares 2-4 mM liposome containing 0.5 \% NDB PC in PBS buffer was recorded in a cuvette. The sample was excited by xenon lamp centred at $485 \mathrm{~nm}$ with $2.5 \mathrm{~nm}$ slit width. A fraction of antibiotics with high concentration in PBS buffer was then introduced to minimize volume change. After mixed well, the spectrum of the sample was measured with the same setting.

\section{Results}

Vancomycin binds to liposome with a millimolar affinity.

We first utilized liposome as membrane mimic to explore if vancomycin associates with the cell membrane at all. We performed isothermal calorimetry experiment by titrating antibiotics into liposome extruded through polycarbonate membrane $(0.1 \mu \mathrm{m}$ diameter). We found that vancomycin, but not $\beta$ lactam antibiotic nafcillin, introduced significant amount of heat change in liposome prepared from a mixture of POPE, POPG and cardiolipin (CL) (75:20:5) (Figure 1), a model mimic for bacterial membrane [17]. Fitting of the data into one-site model gave rise to millimolar level affinity (dissociate constant, $K_{\mathrm{d}}$ ), suggesting that vancomycin weakly, but definitely interact with liposome. The observation is in line with previous studies, where weak binding of vancomycin to model membrane were assessed by ITC [18] and surface plasmon resonance [19].

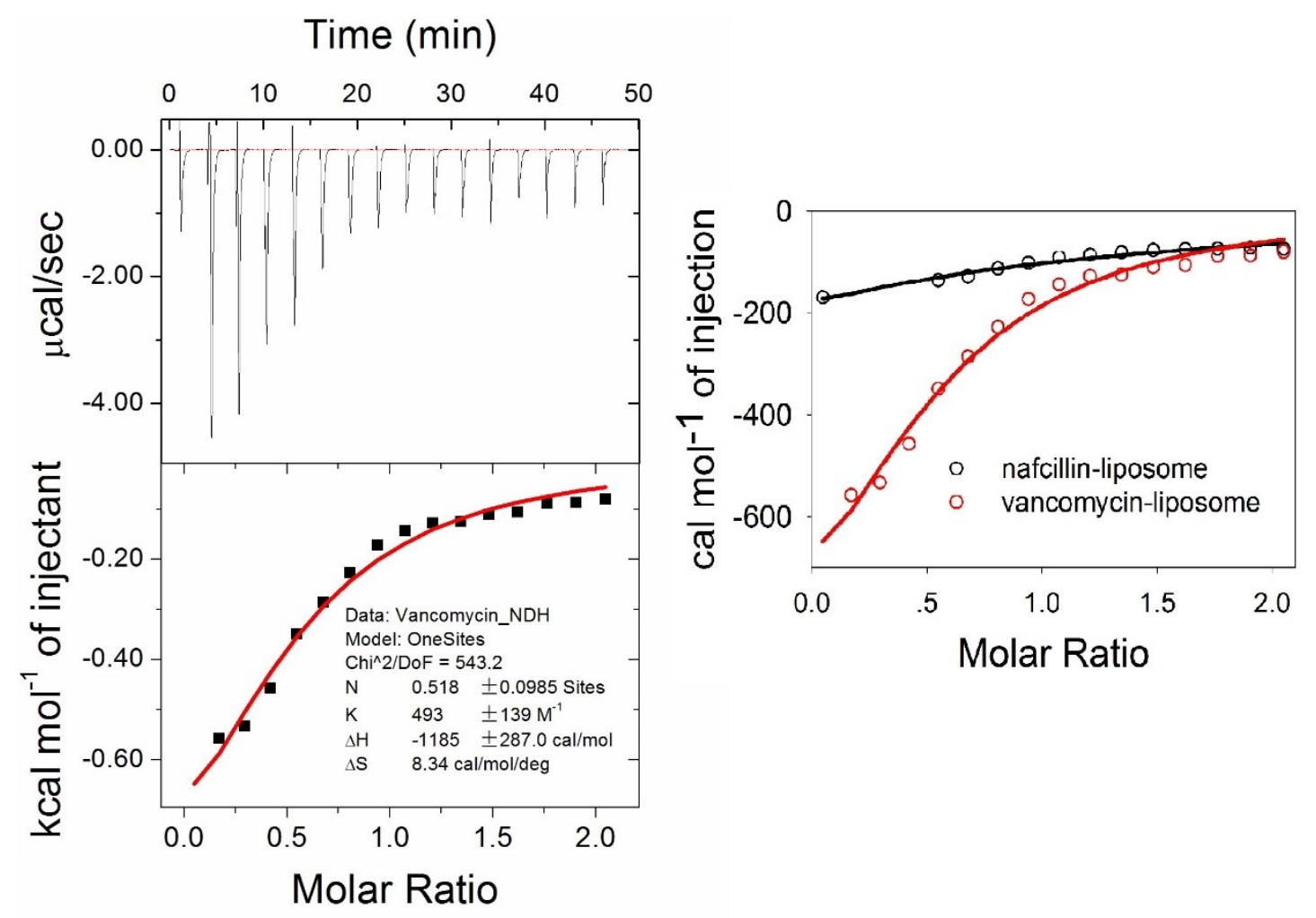

Figure 1. Titration of vancomycin into liposome. Raw data and fitting curve of ITC experiment (left); comparative fitting plot of naficillin and vancomycin titration with liposome (right). 
Vancomycin binds to vesicle derived from Staphylococcus aureus membrane.

Now that we showed vancomycin association with membrane mimic liposome, it is tempting to ask whether the antibiotic can also bind to vesicle derived from biological sample. We thus isolated membrane from gram-positive bacterium Staphylococcus aureus by means of enzymatic digestion, mechanical disruption and syringe needle homogenization [16]. Resultant vesicle was then undergone calorimetric titration with vancomycin as the titrant. According to the fitting result of ITC data, vancomycin also bound to the bacterial derived vesicle (Figure 2), but with much higher affinity (sub-millimolar) than that of liposome (Figure 2). Although we cannot completely rule out the possibility of residual peptidoglycan remaining on the membrane, lysozyme digestion step of the cells should remove most, if not all of them. Apparently, vancomycin binds to the bacterial membrane derived vesicle with higher affinity.
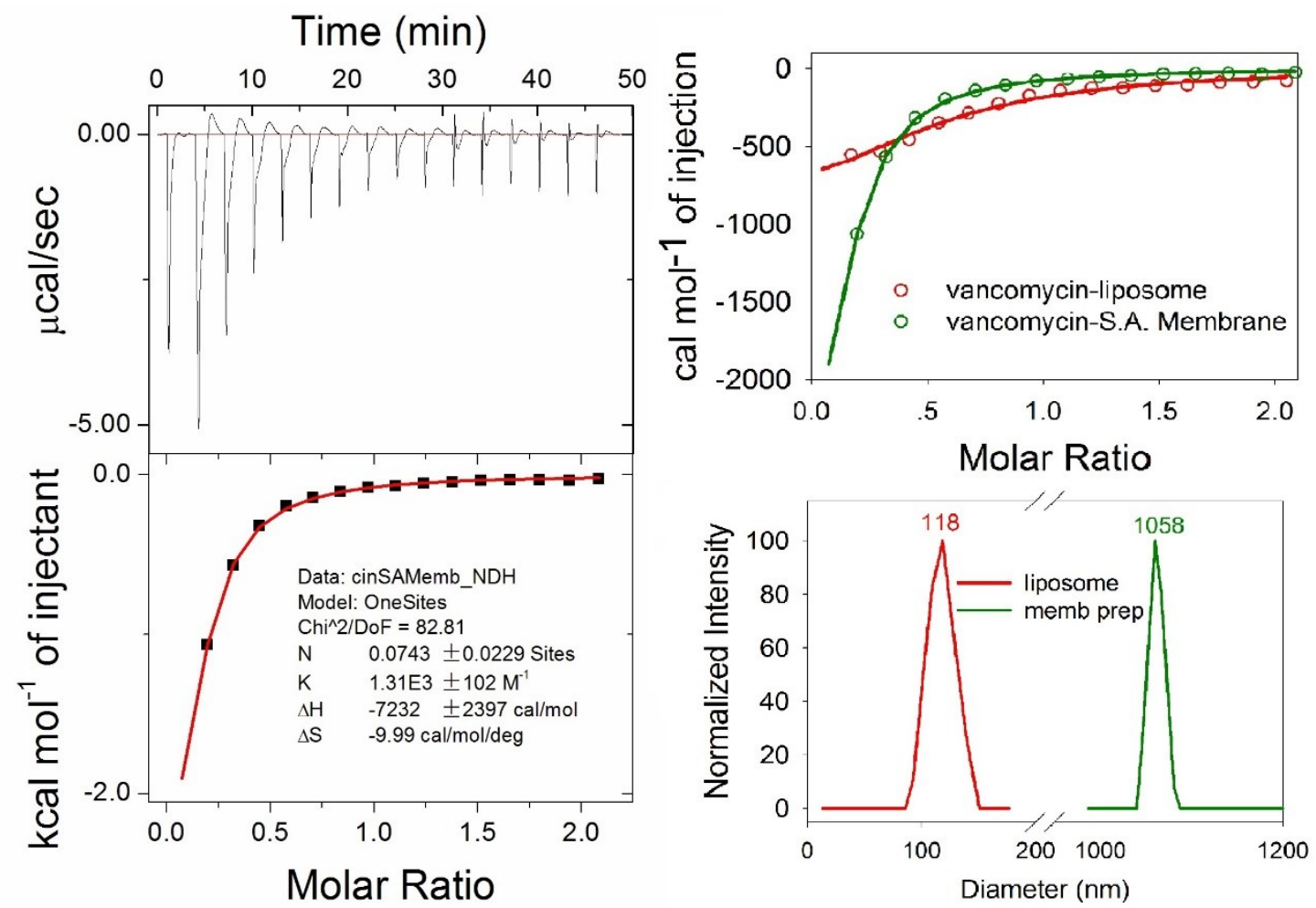

Figure 2. Titration of vancomycin into vesicle derived from Staphylococcus aureus membrane. Raw data and fitting curve of ITC experiment (left); comparative fitting plot of vancomycin titration with liposome and vesicle from membrane (top right); size distribution of liposome and membrane vesicle determined by dynamic light scattering (bottom right).

Vancomycin strengthens the association with liposome as its size increases.

In light of differential binding between liposome and membrane-derived vesicle to vancomycin and the fact that the later measured much larger in diameter than the former (Figure 2), we speculated that the size of liposome/vesicle might play a role in the antibiotic association with membrane mimic. To test the hypothesis, we performed serial calorimetric titration of vancomycin into liposome prepared with polycarbonate membranes of various sizes $(0.1,0.4$ and $1 \mu \mathrm{m})$. We found that the binding affinity of vancomycin increased along with liposome size increment. (Figure 3 ), which indicated that cell surface landscape or/and membrane curvature could be an important factor in vancomycin association. 

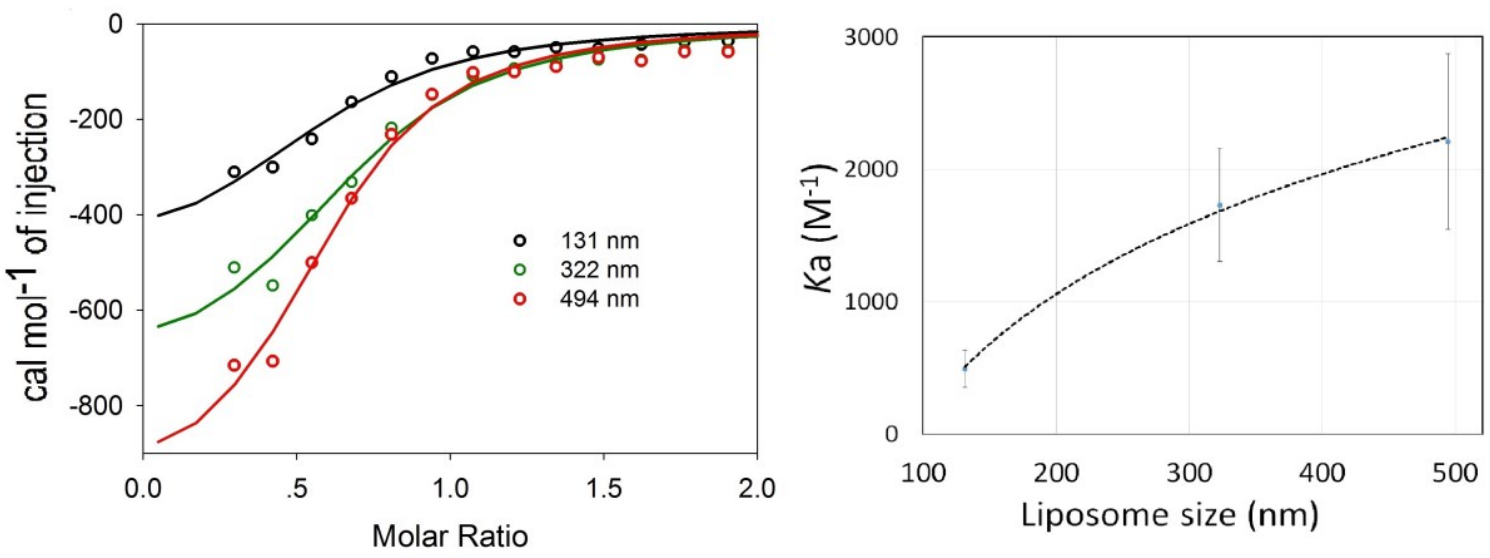

Figure 3. Size-dependent binding of liposome with vancomycin. Data and fitting curve from titrations of liposome were plotted in same figure for comparison (left); $K_{\mathrm{a}}$ value derived from fitting were plotted against the sizes of the liposome (right).

\section{Vancomycin binds preferentially to POPG over cardiolipin and POPE.}

Because the lipids used in the study are similar structurally in fatty acid moiety but vary in head groups, we reasoned that their difference in head group could contribute differently to the association with vancomycin. To explore binding preference of vancomycin among lipids, we performed ITC measurement of the antibiotic with liposomes prepared from individual lipid. Based on the $K_{\mathrm{a}}$ value derived from data fitting, binding affinity of vancomycin to POPG is significantly higher than that to POPE and CL (Figure 4), suggesting that POPG is the major contributor to the association of vancomycin with liposome. The observation could be explained by careful examination of the structure of vancomycin $[20,21]$ and the lipids (Figure 4). Positive charged amine in the head group of POPE does not appear to favour the interaction with uncharged vancomycin. Moreover, embedded hydroxyl group on hydrocarbon chain within two phosphoryl group is unlikely to facilitate binding in the case of cardiolipin. On the other hand, the two hydroxyls on the head group of the POPG could potentially interact with vancomycin via hydrogen bond formation, which promotes the glycopeptide association with lipid membrane [22].

Vancomycin interaction with liposome does not involve acyl terminus of the lipids.

We have shown that vancomycin associates with liposome through its interaction with the head group of the lipids. But it remains unclear if the antibiotic could introduce any change inside the lipid bilayer. Fluorescence spectroscopy was performed to gain more insight into the binding of vancomycin to liposome. We incorporated trace amount $(0.5 \%)$ of tail fluorescence-labeled lipid (NDB-PC) into liposome. Addition of stoichiometric amount of vancomycin did not induce significant change in the spectrum of the liposome (Figure 5), suggesting that binding of vancomycin to liposome might not involve the acyl terminus of the lipid. In other words, it is unlikely for the antibiotic to insert deep into the lipids bilayer. Otherwise, alteration in fluorescence intensity or/and peak position shift would be obvious because of fluorophore disturbing by the insertion, as seen in a control experiment, where pore formation by depsipeptide antibiotics, valinomycin $[23,24]$ reduced liposome fluorescence intensity substantially. This is consistent with the fact that vancomycin and other glycopeptides generally exhibit low membrane permeability $[3,22]$. 
Time (min)

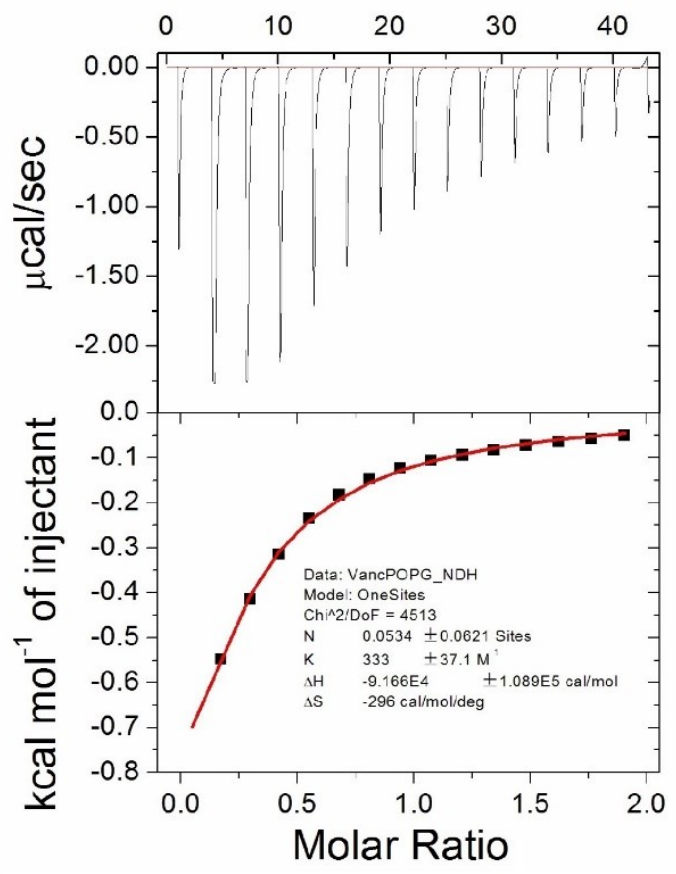

Time (min)

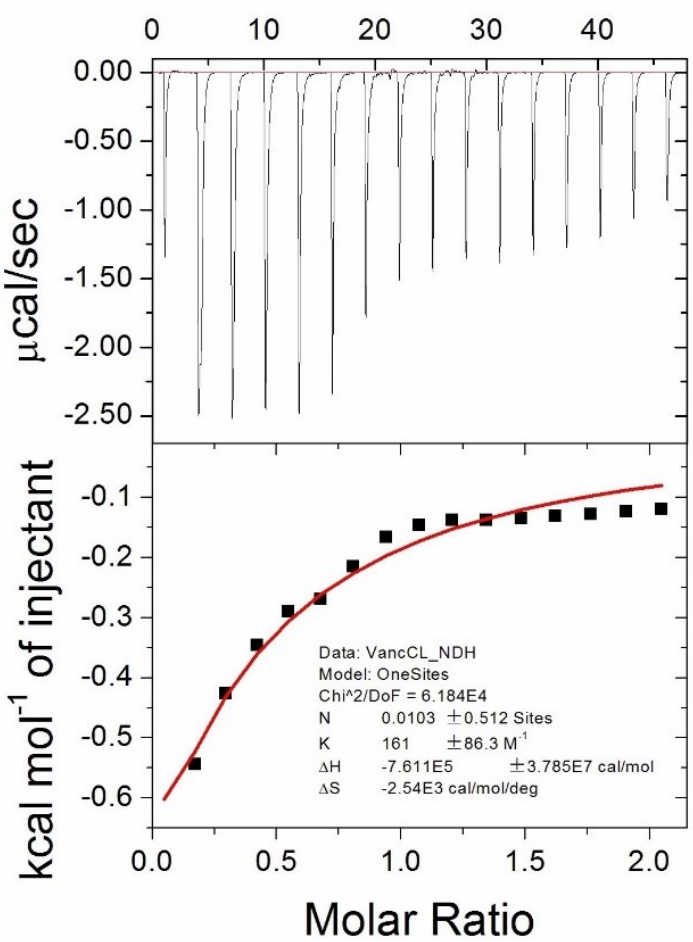

Time (min)
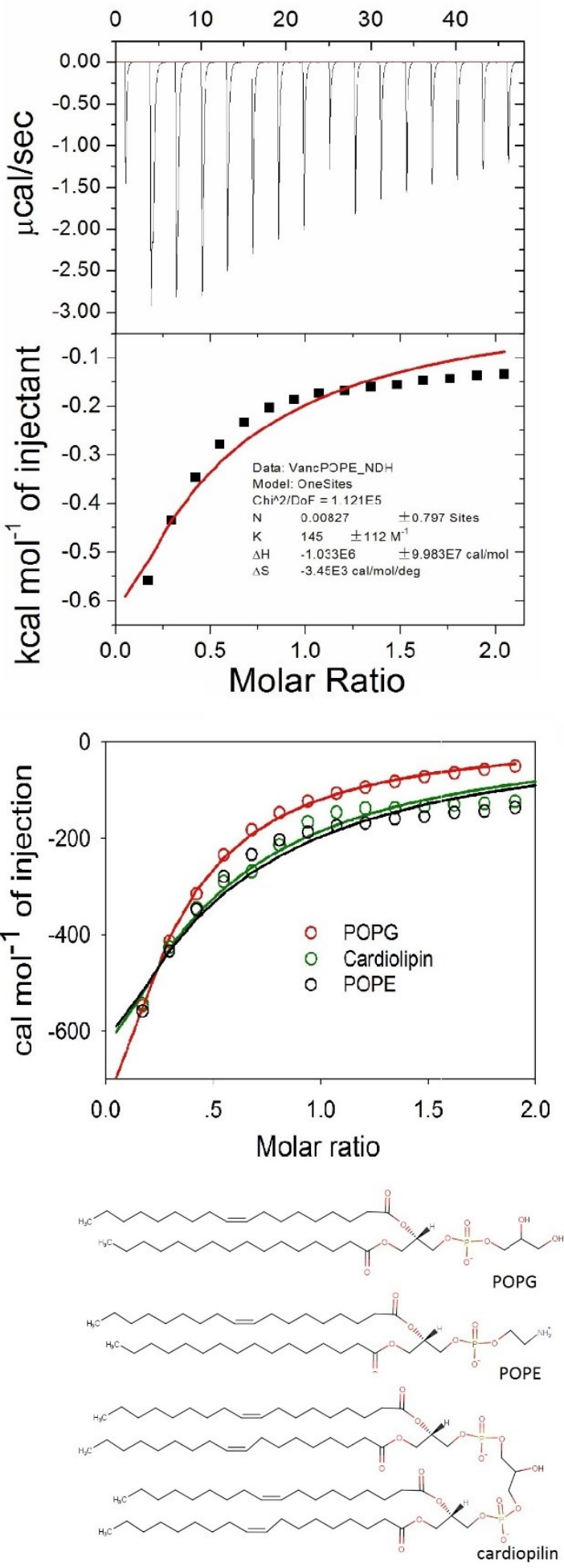

Figure 4. Vancomycin binds preferentially to POPG over POPE and cardiolipin. ITC raw data and fitting of vancomycin titration with POPG (top left), POPE (top right) and cardiolipin (bottom left); Fitting of titrations were plotted for comparison (middle left); Structure of three lipids were shown (bottom right).

\section{Discussion}

We have shown that glycopeptide antibiotics vancomycin associate with biomimetic membrane, a fact have been largely ignored in the field because of its weak interaction $[18,19]$. Furthermore, we showed for the first time that it binds to membrane vesicle derived from gram-positive bacteria Staphylococcus aureus 
in a similar fashion. These observations could potentially open a new avenue for the prototype antibiotics to tackle infectious microbes by blocking cell wall synthesis and interfering cell membrane function simultaneously. Multiple mechanism of action is thought to be an effective strategy to combat with prevalent resistance nowadays rising among pathological microorganism [14,15,25].
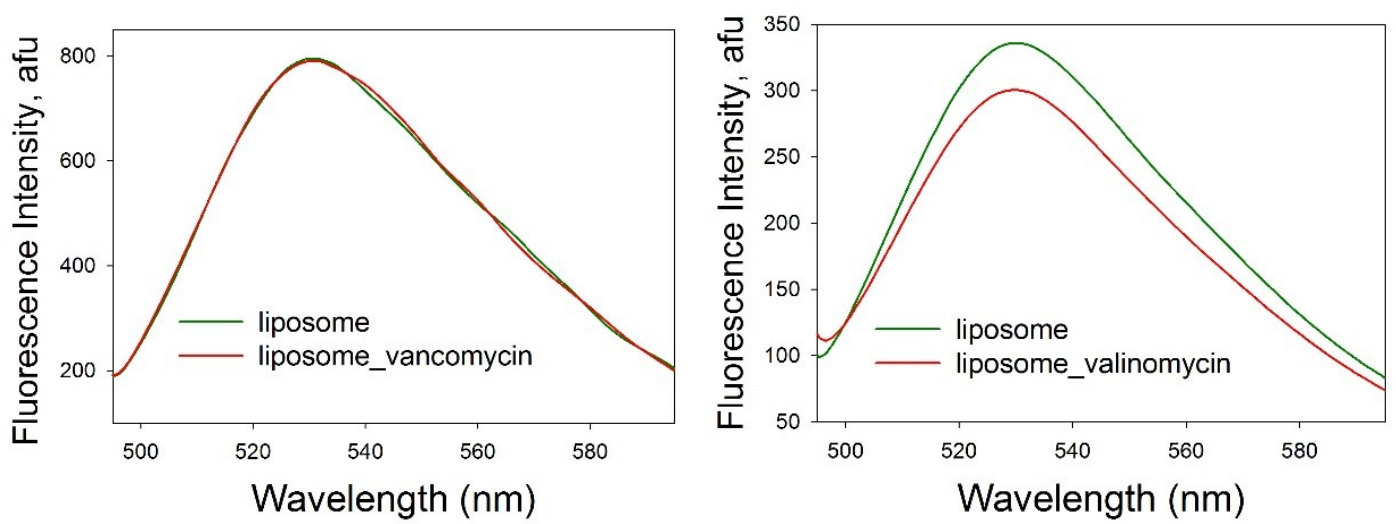

Figure 5. Fluorescence spectra of liposome with fluorescent label. Left panel, spectra of $3.5 \mathrm{mM}$ liposome containing $0.5 \%$ NDB PC in the absence (green line) and presence (red line) of $5 \mathrm{mM}$ vancomycin; right panel, spectra of $1.5 \mathrm{mM}$ liposome containing $0.5 \%$ NDB PC in the absence (green line) and presence (red line) of $2 \mathrm{mM}$ valinomycin.

We also found that vancomycin appears to bind to the membrane from outside and the extent of association depends much on local phospholipid composition and the curvature of the membrane. POPG appears to be the favourite target for vancomycin binding. Moreover larger size (flattened surface) of the liposome enhances the binding. These findings could provide some design ideas for further modification of the antibiotics to optimize its binding to bacterial membrane, which may improve antibacterial potency. To this end, a further investigation of vancomycin structure and bacterial membrane composition is required. In addition, self $[20,21]$ and ligand-induced dimerization [26,27] of vancomycin might also play an important role as a larger hydrophobic interface once shielded by dimeric interaction has to be covered by membrane association.

\section{Conclusions}

We have studied the binding of vancomycin to biomimetic membranes based on three phospholipid components and bacterial membranes by using a variety of biophysical methods, including isothermal calorimetry and fluorescence photometry. It has been shown that the ways of association of vancomycin to biomimetic membranes is very similar to that of vesicle derived from Staphylococcus aureus membrane. Our results clearly indicated that vancomycin associates to the cell membrane from outside, and does not permeate through the cell membrane, which is consistent with the mechanisms of the drug action. By varying the size and composition of the biomimetic membrane vesicles, it has been demonstrated that the extent of association depends on specific type of lipids and curvature of local membrane structure. These observations could potentially open a new avenue to revive the prototype antibiotic to fight infectious microbe through the design of specific tight binders to the outer surface of bacterial membranes to impede cell wall synthesis. 
Acknowledgements: We thank the financial support from the University of Macau (grant no.: SRG201300055-FHS). We thank Prof Jun Zheng (University of Macau) for providing a bacteria strain of Staphylococcus aureus.

\section{References}

[1] L.L. Silver. Challenges of antibacterial discovery. Clin. Microbiol. Rev., 24 (2011) 71-109.

[2] M.A. Fischbach, C.T. Walsh. Antibiotics for emerging pathogens. Science, 325 (2009) 1089-93.

[3] M.A. Kohanski, D.J. Dwyer, J.J. Collins. How antibiotics kill bacteria: from targets to networks. Nat. Rev. Microbiol., 8 (2010) 423-35.

[4] M. Zasloff. Antimicrobial peptides of multicellular organisms. Nature, 415 (2002) 389-395.

[5] K.A. Brogden. Antimicrobial peptides: pore formers or metabolic inhibitors in bacteria?. Nat. Rev. Microbiol., 3 (2005) 238-250.

[6] T. Velkov, P.E. Thompson, R.L. Nation, J. Li. Structure-activity relationships of polymyxin antibiotics. J. Med. Chem., 53 (2010) 1898-1916.

[7] S. Mukherjee, H. Zheng, M.G. Derebe, K.M. Callenberg, C.L. Partch, D. Rollins, D.C. Propheter, J. Rizo, M. Grabe, Q.-X. Jiang, L. V Hooper. Antibacterial membrane attack by a pore-forming intestinal C-type lectin. Nature, 505 (2014) 103-7.

[8] D. Kahne, C. Leimkuhler, W. Lu, C. Walsh. Glycopeptide and lipoglycopeptide antibiotics. Chem. Rev., 105 (2005) 425-448.

[9] D.L. Stevens. The role of vancomycin in the treatment paradigm. Clin. Infect. Dis., 42 Suppl 1 (2006) S51-7.

[10] E. Breukink, B. de Kruijff. Lipid II as a target for antibiotics. Nat. Rev. Drug Discov., 5 (2006) 321332.

[11] U.S. Eggert, N. Ruiz, B. V Falcone, A.A. Branstrom, R.C. Goldman, T.J. Silhavy, D. Kahne. Genetic basis for activity differences between vancomycin and glycolipid derivatives of vancomycin. Science, 294 (2001) 361-364.

[12] G.D. Wright, C.T. Walsh. D-Alanyl-D-alanine Ligases and the Molecular Mechanism of Vancomycin Resistance. Acc. Chem. Res., 25 (1992) 468-473.

[13] L. Otvos, C. Snyder, B. Condie, P. Bulet, J.D. Wade. Chimeric Antimicrobial Peptides Exhibit Multiple Modes of Action. Int. J. Pept. Res. Ther., 11 (2005) 29-42.

[14] V. Yarlagadda, S. Samaddar, K. Paramanandham, B.R. Shome, J. Haldar. Membrane Disruption and Enhanced Inhibition of Cell-Wall Biosynthesis: A Synergistic Approach to Tackle VancomycinResistant Bacteria. Angew. Chem. Int. Ed. Engl., 54 (2015) 13644-13649.

[15] F. Guilhelmelli, N. Vilela, P. Albuquerque, L. da S. Derengowski, I. Silva-Pereira, C.M. Kyaw. Antibiotic development challenges: the various mechanisms of action of antimicrobial peptides and of bacterial resistance. Front. Microbiol., 4 (2013) 353.

[16] R.K. Poole. The isolation of membranes from bacteria. Methods Mol. Biol., 19 (1993) 109-122.

[17] J.K.L. and E.J.P. Mary T. Le. Biomimetic Model Membrane Systems Serve as Increasingly Valuable in Vitro Tools. in: A. George (Ed.), Adv. Biomimetics, InTech, 2011.

[18] S. Al-Kaddah, K. Reder-Christ, G. Klocek, I. Wiedemann, M. Brunschweiger, G. Bendas. Analysis of membrane interactions of antibiotic peptides using ITC and biosensor measurements. Biophys. Chem., 152 (2010) 145-152.

[19] H. Kinouchi, H. Arimoto, K. Nishiguchi, M. Oka, H. Maki, H. Kitagawa, H. Kamimori. Binding Properties of Antimicrobial Agents to Lipid Membranes Using Surface Plasmon Resonance. Biol. Pharm. Bull., 37 (2014) 1383-1389.

[20] Z. Jia, M.L. O'Mara, J. Zuegg, M.A. Cooper, A.E. Mark. Vancomycin: ligand recognition, dimerization and super-complex formation. FEBS J., 280 (2013) 1294-1307. 
[21] P.J. Loll, A.E. Bevivino, B.D. Korty, P.H. Axelsen. Simultaneous recognition of a carboxylatecontaining ligand and an intramolecular surrogate ligand in the crystal structure of an asymmetric vancomycin dimer. J. Am. Chem. Soc., 119 (1997) 1516-1522.

[22] O. Domenech, G. Francius, P.M. Tulkens, F. Van Bambeke, Y. Dufrêne, M.-P. Mingeot-Leclercq. Interactions of oritavancin, a new lipoglycopeptide derived from vancomycin, with phospholipid bilayers: Effect on membrane permeability and nanoscale lipid membrane organization. Biochim. Biophys. Acta - Biomembr., 1788 (2009) 1832-1840.

[23] T.E. Andreoli. The Effect of Valinomycin on the Ionic Permeability of Thin Lipid Membranes. J. Gen. Physiol., 50 (1967) 2527-2545.

[24] P. Bhattacharyya, W. Epstein, S. Silver. Valinomycin-Induced Uptake of Potassium in Membrane Vesicles from Escherichia coli. Proc. Natl. Acad. Sci., 68 (1971) 1488-1492.

[25] S. Gardete, A. Tomasz. Mechanisms of vancomycin resistance in Staphylococcus aureus. J. Clin. Invest., 124 (2014) 2836-2840.

[26] D.McPhail, A. Cooper, S. J. Hammond, J. P. Mackay, M. S. Westwell, D. A. Beauregard, M. F. Cristofaro. Thermodynamics and kinetics of dissociation of ligand-induced dimers of vancomycin antibiotics. J. Chem. Soc. Faraday Trans. 93 (1997) 2283-2289.

[27] M. Rekharsky, D. Hesek, M. Lee, S.O. Meroueh, Y. Inoue, S. Mobashery. Thermodynamics of Interactions of Vancomycin and Synthetic Surrogates of Bacterial Cell Wall. J. Am. Chem. Soc. 128 (2006) 7736-7737.

C 2017 by the authors; licensee IAPC, Zagreb, Croatia. This article is an open-access article distributed under the terms and conditions of the Creative Commons Attribution license (http://creativecommons.org/licenses/by/3.0/) (cc)) EY 